\title{
A New Method for Graph Queries Processing without Index Reconstruction on Dynamic Graph Databases
}

\author{
Hamed Dinari \\ Web and Search Engine Laboratory, School of Computer Engineering, Iran University of Science and Technology \\ (IUST), Narmak, Tehran, Iran \\ Email: dinari@comp.iust.ac.ir, dinari.hamed@yahoo.com
}

\begin{abstract}
Graphs play notable role in daily life. For instance, they are used in variety fields such as social networks, malware detection, and biological networks. Graph data processing performed to extract useful information is known as graph mining. A critical field of graph mining is graph containment problem, in which all graphs containing the query are returned by a graph query $q$. Scanning the whole database (graph query as a subgraph) for a query is a time consuming process. To improve query performance, an inverted index is constructed on the graph database and then the query is performed based on the query. The problem in this process is that when a graph is added to or removed from a database, the inverted index must be reconstructed. The present study proposes a method in which index updating is not needed upon a change in the database. This feature enables simultaneous inverted index updating and querying. The assessment results showed optimum and satisfactory performance of the proposed method.
\end{abstract}

Index Terms - Graph query processing, Graph mining, Data mining, Dynamic graph database.

\section{INTRODUCTION}

Graph is a general data structure used to model complicated and schema less structures. For instance, utilization of graph can be seen in areas such as food chain, UML diagram, software engineering, social networks [1,2,3], ERD diagrams in database [4], gas, water, electricity, and communication, telephone, technology transportation networks, and XML documents $[\mathbf{5 , 6 , 7 ]}$. Graph database system is a database system used to manage graph data. The action of searching a database for preferred models is known as graph mining $[\mathbf{8 , 9}, \mathbf{1 0}, \mathbf{1 1}]$. Given the wide range of graph mining applications (e.g. detecting anomalies in network [12], Internet links analysis [13, 14], graph query indexing $[8,12,13,14]$, and medicine $[\mathbf{1 5}, \mathbf{1 6}])$, great deal of research works have been done on graphs. An interesting matter as to graph mining is graph containment problem. For instance, suppose $D=$ $\left\{g_{1}, g_{2}, g_{3}, \ldots, g_{n}\right\}$ be a graph database with $n$ independent graphs, then query $q$ needs to search all graphs in the database. One of early solutions to do this is to test the whole database with regard to isomorphism, which is not effective as with increase the number of graphs, demand for memory and response time increase exponentially. To deal with this, new methods tried to conduct the query in two steps.

1. Index Construction: the database is scanned and a set of features needed to determine and prune a portion of the database where the answer of query probably is not there is determined. The closer the set of the candidates to the final answer, the higher the performance of the query. This step is offline to be ready to response.

2. Candidate Verification: the candidate graphs obtained in the previous stage are tested as to isomorphism and if the graph is contain the query add to answer set. The paper is arranged as follows: firstly related works are described also definition of the terms and concepts of graph mining (which is not recommend for experienced readers). Section three represents architecture of the proposed method in more detail. Section four discusses the results obtained by the proposed method. The last section concludes the article with conclusion and suggestions for future works.

\section{RELATED WORKS}

There have been in [17] paths were used to constructed the index however, demand for memory was not optimum with increase of index volume and the best performance of the proposed method was obtained with small databases. Frequent subtrees [18] were used in [19] to constructed the index and consequently, considerable increase in tree mining was obtained. Still, the proposed method had the disadvantage of failing to prune the search space as the tree structure is simpler than graph. Frequent subgraphs were used in [20] and closed frequent subgraphs [21] were used in [4] to construct the index. Their results show that considerable decrease in query time was obtained when the query was indexed. On the other hand, accurate query processing method failed to function properly with large databases, which led to 
introduction of similar graph queries [8]. The one challenge faced with by all these methods is that the index must be reconstructed when the database is updated and index construction is time consuming. The proposed method in this paper suits both static and dynamic databases. The main idea is to use Positional Inverted Index (P.I.I) used by search engines. The proposed method relies on hashing technique to implement and store required features, which gives the inverted index opportunity to be updated. In addition, the index can be updated as fast as possible when the graph database is changing, which avoids necessity of totally index reconstruction. Regarding query processing is parallelizable, multithread and vertex invariant techniques are employed to improve checking the candidates [22]. These techniques improve speed of isomorphism test on the subgraphs.

Definition 1. Dynamic graph: A graph in which the edges are added or removed over time so that its structure changes permanently.

Definition 2. Transactional graph database: The data are represented as a set of several independent graphs (e.g. protein, amino acids, and chemical/biological informatics databases). Fig. 1. illustrates a graph database comprised of 4 graphs.

Definition 3. Query processing: Assume $D=$ $\left\{g_{1}, g_{2}, \ldots, g_{N}\right\}$ as a graph database includes $N$ graphs, then a graph query processing is defined: a graph query $q$ is given, all $g i \in D$, where $g_{i}$ is a subgraph of $q$, are returned as a member of answer set. Fig 1. illustrates a graph database and Fig 1d. represents a graph query that returns $\{a, b\}$ as the answer set.

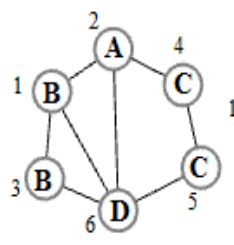

(a)

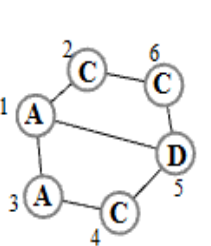

(b)

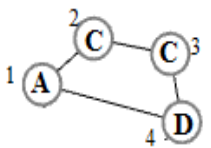

(d)

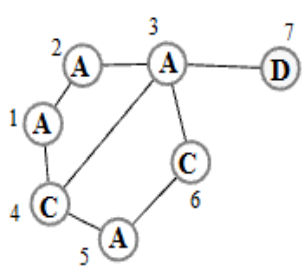

(c)

\section{Proposed Method}

The proposed method for graph query processing is comprised of two parts:

\section{A. Index construction}

\section{B. Query processing}

The index is used to prune part of the search space that does not contain the query answer. To construct the index, the graph database is scanned and, then, a set of features of the database is extracted and stored. The main idea have to use the well-known positional inverted index used in search engines (Fig 2). In short, $t_{1}, t_{2}, t_{3}, \ldots, t_{n}$ are the key terms obtained from the graph database. To accelerate obtaining the key terms, hashing technique was used. Each node in the positing list, implemented using linked list, is comprised of two parts: 1. doc - ID that represents graph number $(\mathrm{No})$, and 2. Position that represents frequency of each edge and other features of the graph. Two hashing tables were used here to improve access to the features, one to store the edges and another to store neighborhood of each node. In addition, columnbased technique (when key/value technique in a hashing table is not the answer to the problem, value section can be implemented as key/value) was used to implement the tables and multithread and thread pooling techniques were used to improve query processing. Following parts discuss each step of index construction and query processing in detail.

\section{A. Index Construction}

The index construction is capable of being updated in case of change in the graphs of the database. Two hashing tables, implemented using column-based techniques, are used to construct the index. So that, one table constructs an inverted index on the edges and another constructed as inverted index on each node and the neighbors. figure 3 illustrates general architecture of the index. Detailed explanations of each section are brought in what follows.

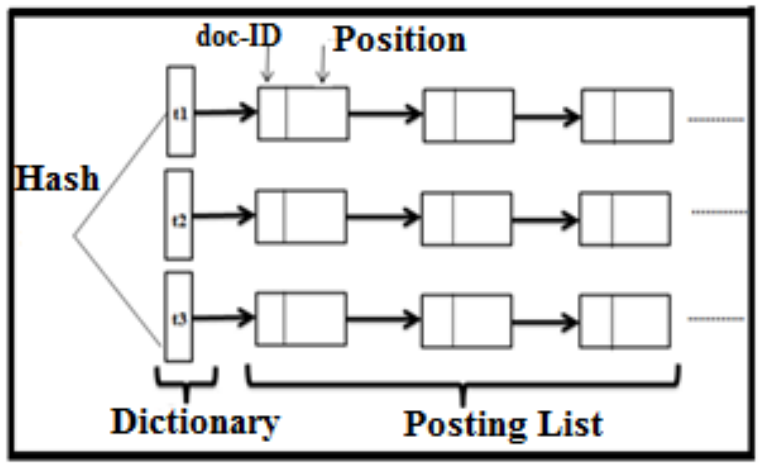

Fig.2. Positional Inverted Index (P.I.I) structure in search engines

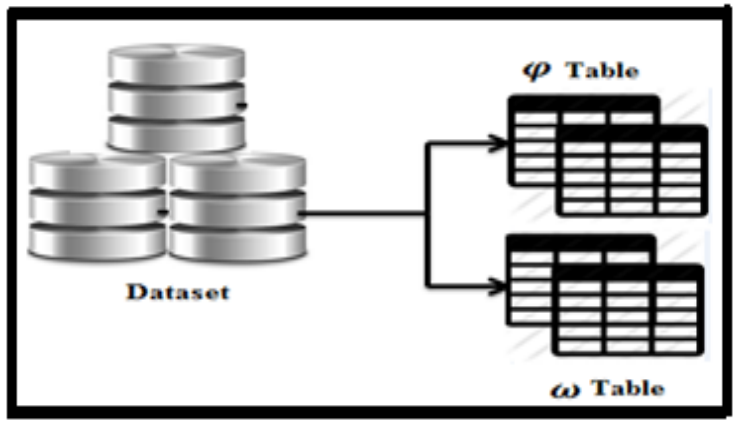

Fig.3. Index construction architecture 


\section{A.1 Construction of Table $\varphi$}

The table is used to store the edges and graph number. The table indicates number of each edge in each graph and number edges of each graph. Fig 4. illustrates a transactional graph database with 2 graphs and table $\varphi$ is filled out like Table 1. according to Fig 4. According to Table 1, edge AB in graph No1 extends between vertex No. 1 and 3 and between vertexes 1 and 4 in graph No 2 . In addition, it is clear that edge AC occurs two times in graph No. 2 between vertexes $1-3$ and $2-3$.

Table 1. Table $\varphi$

\begin{tabular}{|c|c|c|}
\hline Key(Edges) & Key(graph id) & Values(labels) \\
\hline \multirow{2}{*}{ AA } & 1 & 1,2 \\
\cline { 2 - 3 } & 2 & 1,2 \\
\hline \multirow{2}{*}{ AB } & 1 & 1,3 \\
\cline { 2 - 3 } & 2 & 1,4 \\
\hline \multirow{2}{*}{ AC } & 1 & 1,4 \\
\cline { 2 - 3 } & 2 & $1,3 \# 2,3$ \\
\hline \multirow{2}{*}{ BC } & 2 & 3,4 \\
\hline CD & 2 & 3,5 \\
\hline AD & 2 & 1,5 \\
\hline
\end{tabular}

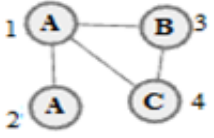

(1)

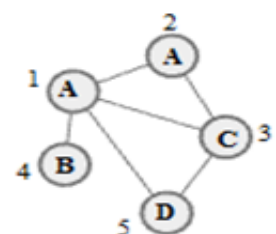

(2)
Fig.4. A transactional graph database with two graphs

\section{A.2 Construction of Table $\omega$}

The table is used to store each node along with its neighbors. An advantage of the table is that it can determine the number of nodes of a graph, frequency of each node in a graph, and number of neighbors of each node. Table 2 indicates how a table $\omega$ is filled out based on database of Fig 4.

Table 2. Table $\omega$

\begin{tabular}{|c|c|c|}
\hline $\begin{array}{c}\text { Key (graph } \\
\text { ids) }\end{array}$ & $\begin{array}{c}\text { Key } \\
\text { (nodes) }\end{array}$ & Value(Neighbors) \\
\hline \multirow{3}{*}{1} & 'A' & 'A'\%'A'\#'B'\#'C' \\
\cline { 2 - 3 } & 'B' & 'A'\#'C' \\
\cline { 2 - 3 } & 'C' & 'A'\#'B' \\
\hline \multirow{3}{*}{2} & 'A' & 'A'\#'B'\#'C'\#'D'\%'A'\#'C' \\
\cline { 2 - 3 } & 'B' & 'A' \\
\cline { 2 - 3 } & 'C' & 'A'\#'A'\#'D' \\
\cline { 2 - 3 } & 'D' & 'A'\#'C' \\
\hline
\end{tabular}

For instance, according to table $\omega$, one may say that graph No.1 has two ' $A$ ' nodes so that one has ' $A$ ' as its neighbor and another has ' $A$ ', ' $B$ ', and ' $C$ ' as its neighbors
(\# is separator of the neighbors of each node). In the case of dynamic graphs, a thread is used to update the tables and a trigger is used to update the database. Algorithm 1 illustrates how the index is constructed.

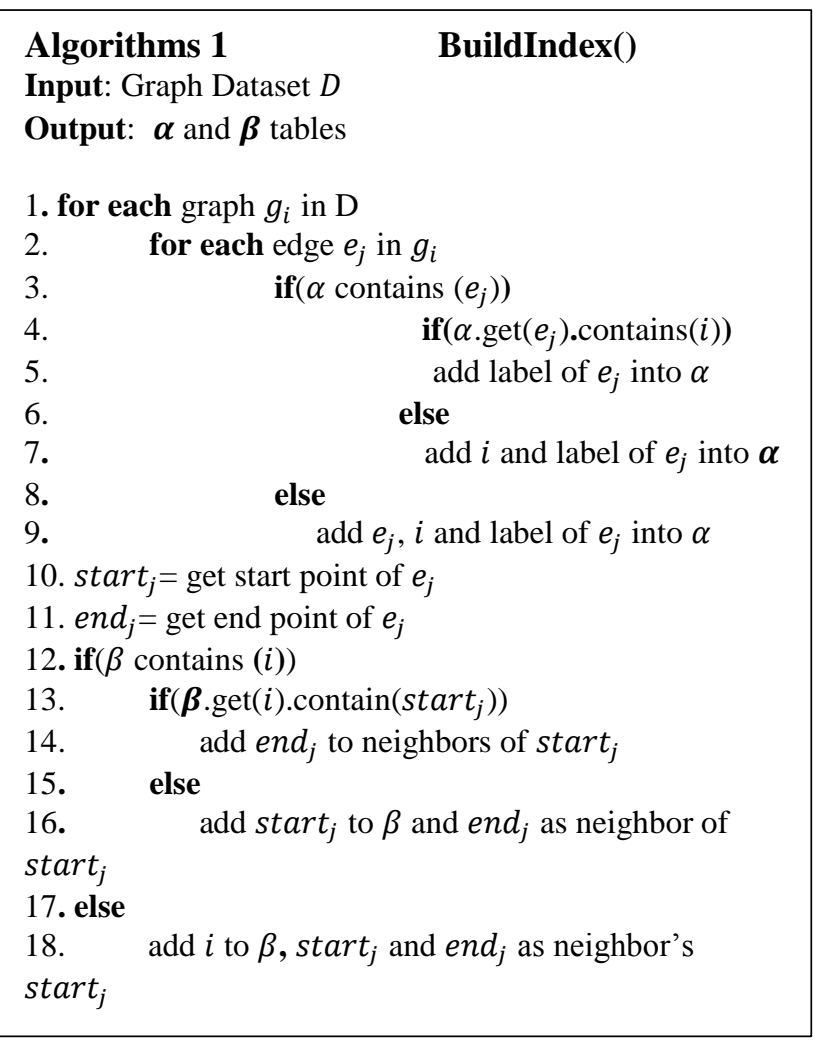

\section{B. Query Processing}

The constructed index is used to prune the search space and improve performance of query processing. General architecture of the query processing is illustrated in Fig 5.

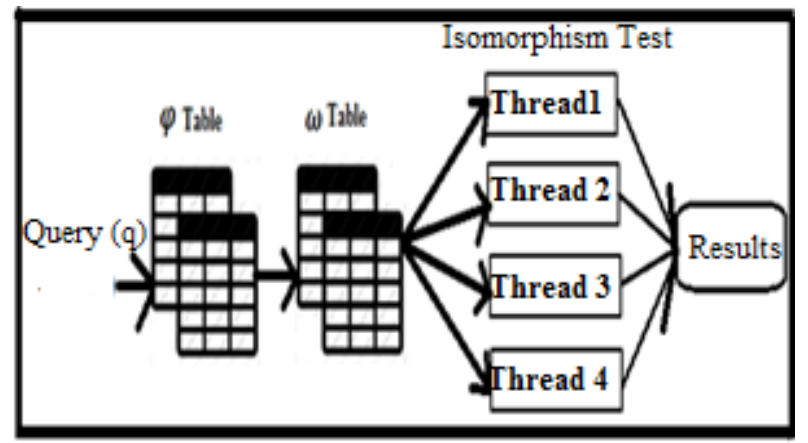

Fig.5. General architecture of query processing

Immediately after placing a query request at the input, frequency of each edge in the query is computed. Afterward, frequency of query edges in the graph database is computed based on the data of Table $\varphi$ and in turn graph-id is obtained. Afterward, the set of the graphs in which the query can be performed is obtained for each element of the obtained set based on table $\omega$ and comparing the nodes of a graph (neighbor of the nodes and their labels) and the query. Finally, isomorphism test 
is carried out on the set of the graphs. Here, multi-threat technique is used so that candidate id-graph set is distributed over threads implemented independently (thread pooling technique) and the threads perform isomorphism test using vertex invariant technique [25]. In general, two threads that are implemented independently are used, one for updating the index (Tables $\omega$ and Table $\varphi$ ) and one for collecting queries from inputs and processing. Processing time is obtained as equation.(1).

$$
T_{\text {response }}=T_{\text {search }}+\left|C_{q}\right| *\left|T_{\text {isoSubG }}\right|
$$

Where, $\mathrm{T}_{\text {search }}$ is the query time between tables $\omega$ and $\varphi,\left|C_{\mathrm{q}}\right|$ is number of candidate graphs that need isomorphism test, and $\mathrm{T}_{\mathrm{isosubG}}$ is the required time of isomorphism test. Query processing algorithm is illustrated in Algorithm 2. In Fig 6 a general architecture of dynamic graph query processing is indicated, when graph database is changed or update (graphs/edges is removed or inserted) a trigger updates the index. Update the index and query processing performed simultaneously.

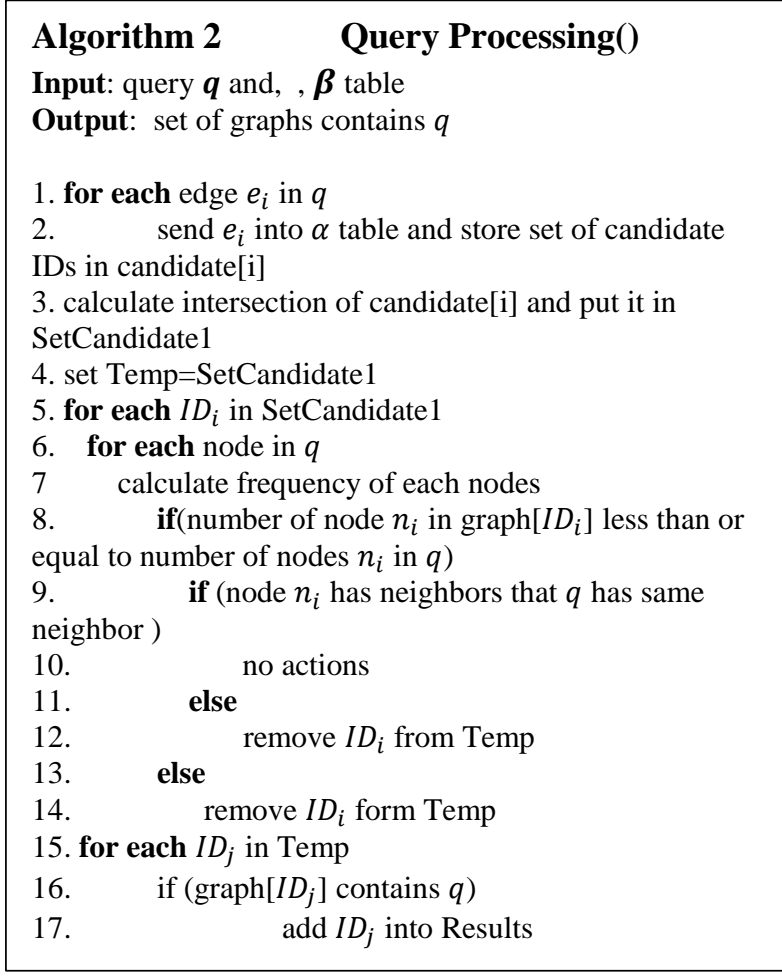

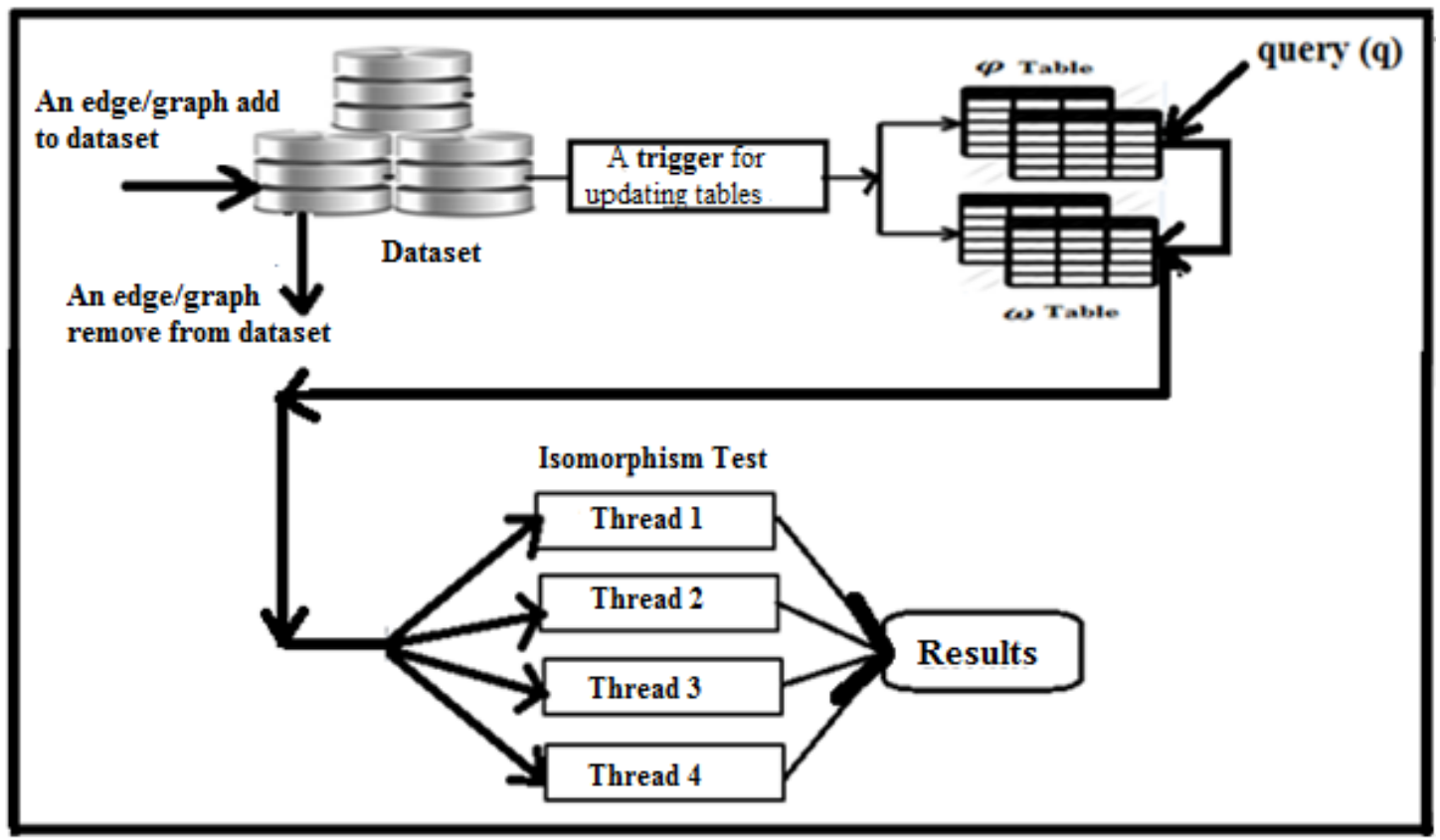

Fig.6. dynamic graph query processing architecture

\section{EXPERIMENTS AND EVALUATION}

The proposed method is implemented in Java, Netbeans IDE6.9, Microsoft Windows 7. The datasets were generated by an algorithm for generating real databases. Fig 7. and Fig 8. illustrate features of the databases. Fig 7. shows the nodes and their frequency and Fig. 8 illustrates the edges and their frequency in different datasets. The datasets features are listed in Tables 3, 4 .

In this paper graph queries processing (graph query as a subgraph) was performed on a transactional graph database .That is because the database is used in different areas; for instance, in the case of "malware detection" $[\mathbf{2 3}, \mathbf{2 4}, \mathbf{2 5}]$ several malware are taken as input. By extracting the APIs from the codes (the malware are run in sandbox or VM (Virtual Machine) environment to 
avoid damages to the system) after retrieval, the APIs of the malware's codes as "control flow graph" is extracted. Then, according to graph mining algorithms such as gspan [18] frequent subgraphs are mined and indexed $[4,19,14]$. The obtained index is used to detect the malware (as a signature). The point here is that, however, when a new malware is introduced, the index needs to be thoroughly updated as the mining algorithm of subgraphs needs to repeat the mining of the frequent subgraphs. This is a serious disadvantage taking into account the rate of generation of malwares. This explains the reason for proposing the method. because the proposed method relies on hashing technique to construct the index, GraphGrep [17] hashing-based technique was selected for comparison. GraphGrep method extracts and stores all the possible paths up to a specific length. Thereby, finding all paths needs considerable time and memory for large transaction graph databases. In addition, when an edge of the graph is removed (e.g. a malware modifies its structure), the previously stored paths must be updated as well (there are many paths in the index including the removed edge). Moreover, by adding new edge to graph, all the possible paths up to a specific length needs to be extracted and added to the index. This brings high demand for memory need to store the index, while updating the index is time consuming. Therefore, it was omitted from the assessments as the databases used for assessment were too large and time consuming for GraphGrep to generate index and answer the queries. On the other hand, the proposed method utilizes a proper data structure based on the idea of search engines so that only each edge of the input graph is read for construct the index. In addition, the index can be updated with time complexity of $\mathrm{O}(1)$ when new graphs are added or removed from the database. On one hand, GraphGrep method uses the paths to prune the state space and on the other hand the paths, which is the simplest data structure, is not effective in pruning the unwanted graphs, which are not part of the query. It also fails to detect closed loops and rings. Candidate verification stage also needs long time to perform isomorphism test. The proposed method uses invariant vertex technique [22] for isomorphism test, which is more effective comparing with previous methods that rely on adjacency matrix for isomorphism test. The reason that the diagrams are pointy at specific values (peak of diagrams) is that degree and label of nodes of the queried graphs are uniform and carrying out isomorph test on the query based increases isomorphism time. However, after distribution, degree and the labels are no longer uniform and thus ,time of isomorphism test is attenuated. Regarding assessments, diagrams are snapshots that are taken at the time of implementation as they are changing permanently. Clearly, the changes on GraphGrep are not displayed as updating the database using GraphGrep is too slow. The point is that GraphGrep is actually designed for static environments and does not suit for dynamic environments.
Table 3. the nodes and their frequencies

\begin{tabular}{|c|c|c|c|c|c|}
\hline \multicolumn{7}{|c|}{ Dataset Size } \\
\hline \multicolumn{2}{|c|}{$\mathbf{1 0 K}$} & $\mathbf{2 0 K}$ & $\mathbf{3 0 K}$ & $\mathbf{4 0 K}$ & $\mathbf{5 0 K}$ \\
\hline Nodes & \#Freq & \#Freq & \#Freq & \#Freq & \#Freq \\
\hline A & 12288 & 24673 & 37019 & 49348 & 61617 \\
\hline G & 12166 & 24491 & 36726 & 49089 & 61407 \\
\hline F & 12279 & 24612 & 37061 & 49455 & 61701 \\
\hline E & 12372 & 24580 & 36874 & 49097 & 61357 \\
\hline D & 12288 & 24329 & 36661 & 49071 & 61513 \\
\hline C & 12355 & 24713 & 36932 & 49177 & 61607 \\
\hline B & 12266 & 24661 & 36793 & 48988 & 61273 \\
\hline
\end{tabular}

Table 4. the edges and their frequency

\begin{tabular}{|c|c|c|c|c|c|}
\hline \multicolumn{7}{|c|}{ Dataset Size } \\
\hline \multicolumn{2}{|c|}{$\mathbf{1 0 K}$} & $\mathbf{2 0 K}$ & $\mathbf{3 0 K}$ & $\mathbf{4 0 K}$ & $\mathbf{5 0 K}$ \\
\hline Edge & \#freq & \#freq & \#freq & \#freq & \#freq \\
\hline EE & 3259 & 6479 & 9679 & 12821 & 16090 \\
\hline BD & 6364 & 12704 & 19094 & 25699 & 32206 \\
\hline BC & 6374 & 12890 & 19116 & 25741 & 32243 \\
\hline BB & 3147 & 6322 & 9465 & 12551 & 15717 \\
\hline CG & 6416 & 12638 & 19034 & 25261 & 31795 \\
\hline CF & 6608 & 13021 & 19483 & 26041 & 32594 \\
\hline FG & 6343 & 12760 & 19172 & 25592 & 32049 \\
\hline FF & 3154 & 6422 & 9674 & 13021 & 16157 \\
\hline CE & 6500 & 12909 & 19162 & 25572 & 31971 \\
\hline CD & 6544 & 12939 & 19521 & 26053 & 32605 \\
\hline CC & 3159 & 6382 & 9518 & 12818 & 16204 \\
\hline AG & 6304 & 12814 & 19212 & 25763 & 32335 \\
\hline AF & 6348 & 12799 & 19308 & 25873 & 32262 \\
\hline DG & 6287 & 12653 & 19094 & 25565 & 32101 \\
\hline GG & 3159 & 6403 & 9629 & 12810 & 16119 \\
\hline AE & 6626 & 12918 & 19358 & 25786 & 32098 \\
\hline DF & 6453 & 12737 & 19205 & 25733 & 32284 \\
\hline AD & 6243 & 12544 & 19029 & 25600 & 32010 \\
\hline DE & 6512 & 12743 & 19219 & 25610 & 32163 \\
\hline AC & 6607 & 13084 & 19528 & 25957 & 32385 \\
\hline DD & 3092 & 6159 & 9391 & 12668 & 15926 \\
\hline AB & 6362 & 12861 & 19264 & 25686 & 32239 \\
\hline AA & 3163 & 6430 & 9678 & 12819 & 16035 \\
\hline BG & 6421 & 12959 & 19312 & 25703 & 32154 \\
\hline BF & 6310 & 12827 & 19221 & 25669 & 32135 \\
\hline EG & 6256 & 12613 & 19010 & 25439 & 31891 \\
\hline EF & 6363 & 12668 & 19151 & 25462 & 31702 \\
\hline BE & 6377 & 12802 & 19065 & 25460 & 31892 \\
\hline & & & & & \\
\hline
\end{tabular}

Fig 7. illustrated index construction time. clearly, increase of database size leads the increase of time. However, the performance of the index construction shows great improvement thanks to hashing technique.

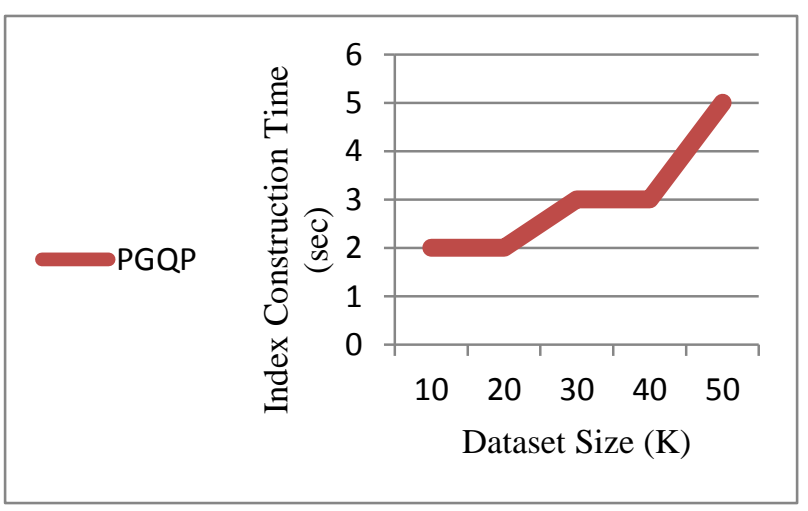

Fig.7. Index construction time 
Fig 8, Fig 9, and Fig 10 illustrates query processing time. All the queries are selected randomly and some of them have equal size though with different structure.

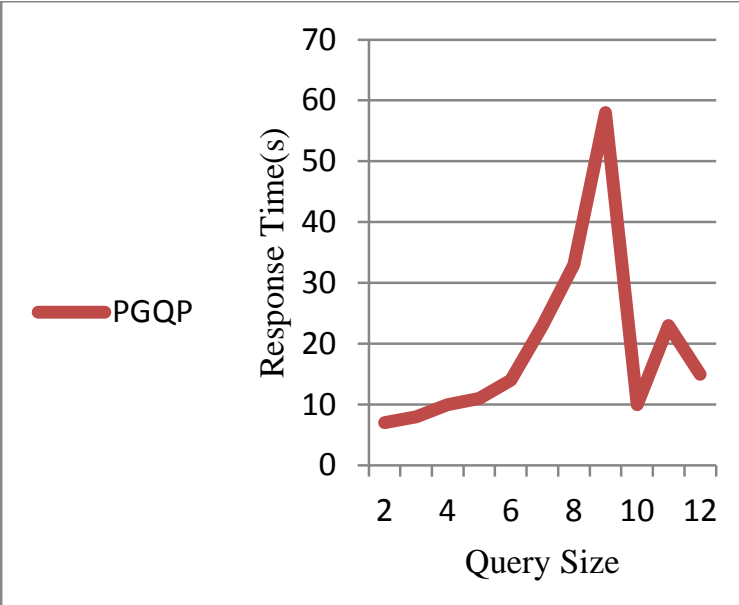

Fig.8. Query processing on (a) 25k left, (b) 30k right

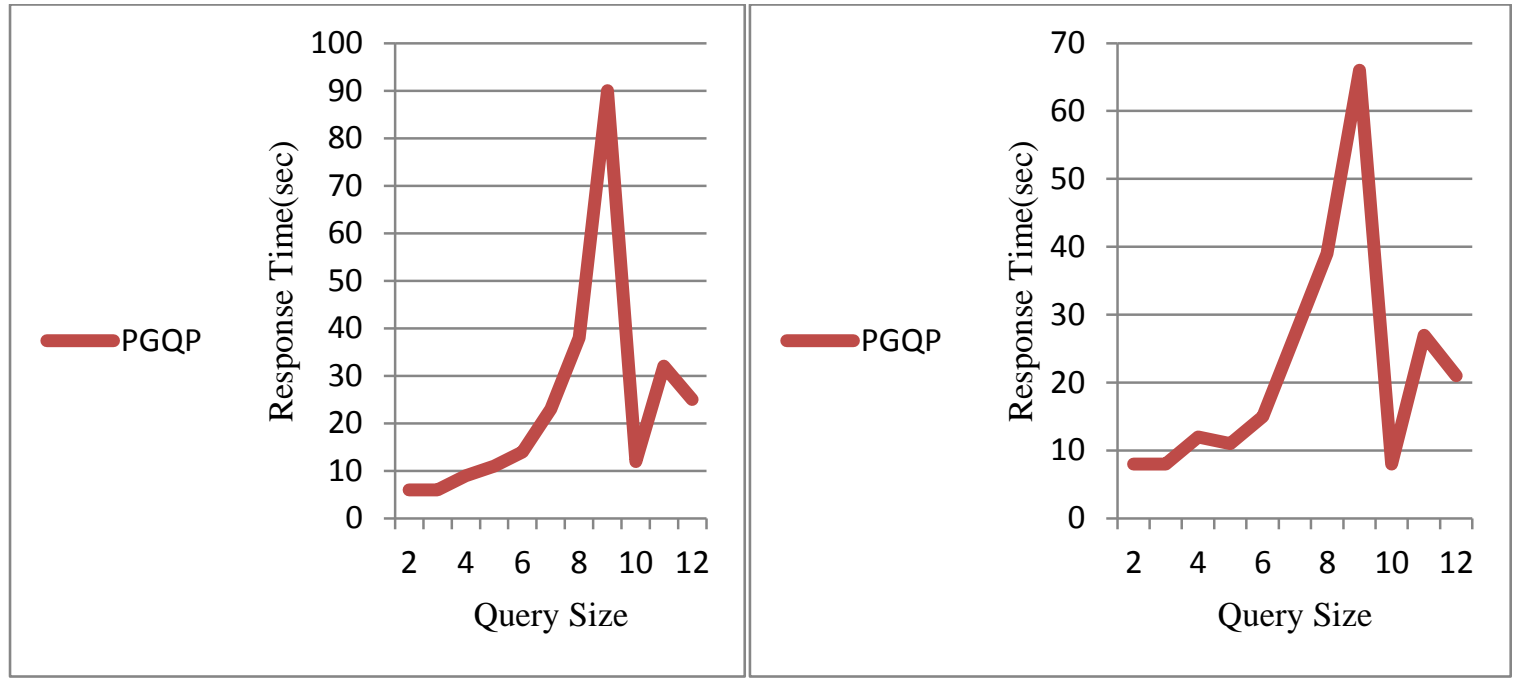

Fig.9. Query processing on (a) 35k left, (b) 40k right

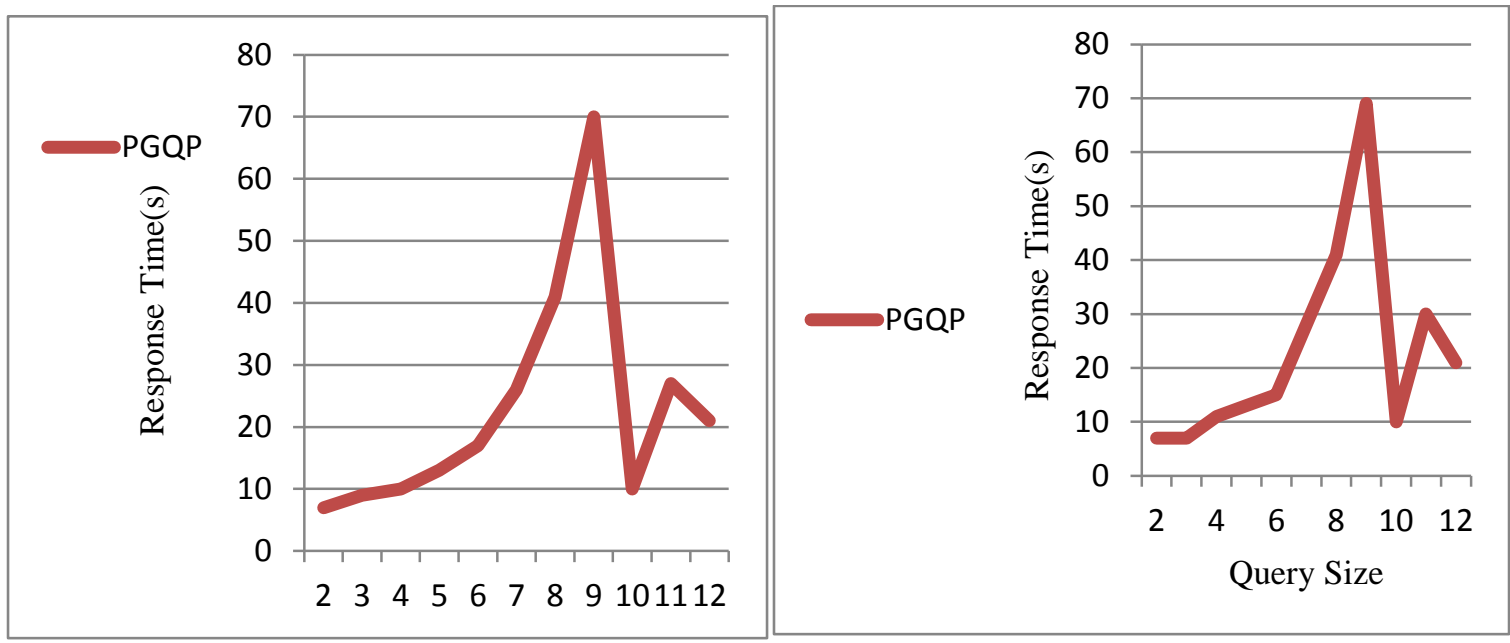

Fig. 10. Query processing on (a) $45 \mathrm{k}$ left, ( b) $50 \mathrm{k}$ right 


\section{CONCLUSION, FUTURE WORKS}

A short introduction to application of graphs was provided first. Given the weight of graph processing regarding graph mining methods, the pertinent concepts and topics were discussed. An obstacle in the way of graph query processing is that the index of database should be reconstructed each time the graph is changed. To solve this, Positional Inverted Index (P.I.I) used by search engines was proposed. So that the index tables can be updated when the database graphs are dynamic with no need to reconstruct the index. This approach led to considerable increase in query processing performance. Taking into account that graph processing have wide range of applications in different fields, the proposed method can the used for designing online malware detection applications. So that by adding a new virus to the dataset, the signature (i.e. index) does not need reconstruction.

\section{REFERENCE}

[1] Wasserman, S., Faust, K., and Iacobucci. "Social network analysis: Methods and applications". Cambridge university Press, 1994.

[2] S.Misra, R.Barthwal, M.S.Obaidat. "Communication Detection in an Integrated Internet of Things and Social Network Architecture" Communication QOS, Reliability and Modeling Syposium, no. IEEE, pp. 2787-2805. 2012.

[3] L.YAN, J.WANG. "Extracting regular behaviors from social media networks," in Third International Conference on Multimedia Information Networking and Security, 2011.

[4] Cheng, James, Y.Ke, W.Ng, and An Lu. "FG-Index: Towards Verification-Free Query Processing on Graph Databases," in international conference on Management of data, Beijing, pp. 857-872, 2007.

[5] Ivancsy,I. Renata, I.Vajk. "Clustering XML documents using frequent subtrees," Advances in Focused Retrieval, vol. 3, pp. 436-445, 2009.

[6] J.Yuan, X.Li, L.Ma. "An Improved XML Document Clustering Using Path Features" in Fifth International Conference on Fuzzy Systems and knowledge Discovery, vol 2, 2008.

[7] Rajaraman, J.D.Ullman. "Mining of Massive Datasets", 2nd editon, 2012.

[8] C.C.Aggarwal,Wang, Haixun. "Managing and Mining Graph Data". Springer, 2010

[9] J.Han, M.Kamber. "Data Mining Concepts and Techniques" USA: Diane Cerra, 2006.

[10] H.dinari, H.naderi. "A survry of frequent subgraphs snd subtree mining methods", International Journal of Computer Science and Business Informatics(IJSCBI), vol. 14(1), 39-57, 2014.

[11] S.Sakr, E.Pardede. "Graph Data Management: Techniques and Applications". United States of America: Information Science Reference (an imprint of IGI Global), 2012.

[12] Peng, Tao, et al "A Graph Indexing Approach for Content-Based Recommendation System" in IEEE Second International Conference on Multimedia and Information Technology (MMIT),pp. 93-97, 2010.
[13] Yildirim, Hilmi, and M.J.Zaki. "Graph indexing for reachability queries", in 26th International Conference on Data Engineering Workshops (ICDEW), pp. 321-324, 2010.

[14] Swati C. Manekar, M.Narnaware. "Indexing Frequent Subgraphs in Large graph Database using Parallelization" International Journal of Science and Research (IJSR),2(no 5), pp. 426-430, 2013.

[15] Ranu, Sayan, and Ambuj K. Singh. "Indexing and mining topological patterns for drug discovery" in Proceedings of the 15th International Conference on Extending Database Technology, pp. 562-565, 2012.

[16] Kramer, S, De Raedt, L, and Helma, C, "Molecular feature mining in HIV data", in In Proc. of the 7th ACM SIGKDD International Conference on Knowledge Discovery and Data Mining (KDD-01),pp.136-143, 2001.

[17] Giugno, Rosalba, and D.Shasha. 2002. "Graphgrep: A fast and universal method for querying graphs," in IEEE Proceedings 16th International Conference on Pattern Recognition, vol. 2, pp. 112-115, 2002.

[18] Yan, Xifeng, and Jiawei Han. "gspan: Graph-based substructure pattern mining" in IEEE International Conference on Data Mining (ICDM), pp. pp. 721-724, 2002.

[19] He, Huahai, Ambuj K. Singh. "Closure-tree: An index structure for graph queries," in 22nd International Conference on Data Engineering (ICDE), pp. 38-48, 2006.

[20] Yan, Xifeng, S.Philip Yu, and J.Han. "Graph indexing: a frequent structure-based approach" in ACM SIGMOD international conference on Management of data(SIGOM), pp. 335-346, 2004.

[21] Yan, Xifeng, X. Zhou, and J.Han."Mining closed relational graphs with connectivity constraints", in ACM SIGKDD international conference on Knowledge discovery in data mining (SIG), pp. 324-333, 2005.

[22] M.Kuramochi, and G.Karypis, and et al. "An efficient algorithm for discovering frequent subgraphs" IEEE Transactions on Knowledge and Data Engineering, vol. 9, pp. 1038-1054, 2004.

[23] Christodorescu, M., Jha, S., Seshia, S. A., Song, \& Bryant. "Semantics-aware malware detection" IEEE Symposium on Security and Privacy, pp. 32-46, 2005

[24] Elhadi, Ammar AE, Mohd A. Maarof, and Ahmed H. Osman."Malware detection based on hybrid signature behaviour application programming interface call graph" American Journal of Applied Sciences, Vol. 9(no.3), 2012.

[25] Hu, Xin, Tzi-cker Chiueh, and Kang G. Shin. "Largescale malware indexing using function-call graphs" in 16th ACM conference on Computer and communications security. pp. 611-620, 2009.

[26] G.XU, Y.zhang, L.li. "Web mining and Social Networking". melbourn: Springer, 2010.

[27] Ko, Calvin. "Logic induction of valid behavior specifications for intrusion detection", in In IEEE Symposium on Security and Privacy (S\&P), pp. 142-155, 2000.

[28] Berendt, Hotho, and Stumme. "semantic web mining," in Conference International Semantic Web (ISWC), pp. 264278, 2002. 


\section{Authors' Profiles}

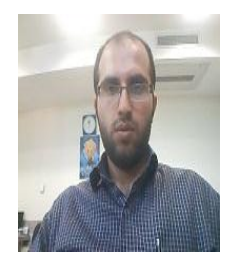

Hamed Dinari received his B.SC. Degree in Computer Engineering (Software) from University of Ilam, Ilam, IRAN, and M.SC. in Computer Engineering (Software) from Iran University of Science and Technology (IUST), Tehran, IRAN, in 2012, 2014 respectively. His research interests are mostly about Database Systems, Data Mining, Graph Mining, and indexing.

How to cite this paper: Hamed Dinari,"A New Method for Graph Queries Processing without Index Reconstruction on Dynamic Graph Databases", International Journal of Modern Education and Computer Science(IJMECS), Vol.9, No.2, pp.47-54, 2017.DOI: 10.5815/ijmecs.2017.02.06 